Ann. Génét. Sél. anim., I980, 12 (4), 383-394.

REVUE

\title{
Le déterminisme génétique de l'hypertrophie musculaire chez le Porc $(*)$
}

\author{
L. OLIIVIER
}

avec la collaboration technique de P. Dando, C. FELgines et Marie-Reine Perretant

Station de Génétique quantitative et appliquée,

Centre national de Recherches zootechniques, I.N.R.A., 78350 Jouy-en-Josas, France

\section{Résumé}

L'apparition du porc belge de Piétrain a suscité très tôt des spéculations quant à son origine Une hypothèse fréquemment avancée par les auteurs belges était que les éleveurs de Piétrain avaient détecté et sélectionné une mutation, car le développement musculaire exceptionnel de cette race pouvait difficilement s'expliquer par une sélection sur un caractère à déterminisme polygénique. Une expérience de croisement réalisée en France il y a une quinzaine d’années suggérait aussi l'existence d'un gène "majeur " responsable de l'hypertrophie musculaire du porc de Piétrain, telle qu'elle peut être jugée d'une part sur l'animal vivant par appréciation visuelle et d'autre part sur la carcasse soumise à une découpe. Enfin, la mise en évidence dans cette même race d'un gène responsable du syndrome d'hyperthermie maligne déclenché par l'inhalation d'halothane et la liaison étroite entre cette sensibilité à l'halothane et le développement musculaire ont conduit naturellement à postuler que le même gène, par une action pléiotropique, est responsable des deux phénomènes. L'inhalation d'halothane peut donc être considérée comme ur test spécifique de l' "entité" Piétrain telle qu'eile avait été précédemment définie. Les relations entre ce gène et des polymorphismes biochimiques à trois locus voisins ont permis de dresser un début de "carte chromosomique " sur une longueur d'environ 6 centimorgans. Les répercussions de ce phénomène sur la qualité de la viande ont fait l'objet de recherches nombreuses et le mécanisme biologique sous-jacent serait unt défaut de la membrane mitochondriale affectant les mouvements de l'ion Calcium. Par contre, le mécanisme biologique induisant l'hypertrophie musculaire reste encore inexpliqué.

\section{Introduction}

La race porcine de Piétrain doit son nom à une commune de la province belge du Brabant où elle apparut vers I920. Mais ce n'est qu'en I950 que la race fut officiellement créée et elle connut dès lors un développement rapide en Belgique

(*) Communication présentée au séminaire "Hvpertrophie musculaire chez les bovins " organisé par la C.E.E. à Toulouse, IO-I 2 juin I980. 
et plus tard dans d'autres pays européens, dont la France qui l'importa en I957. Quant à l'origine de la race, WIL,EMS (I960) fait remarquer, en citant un rapport de DERWA, que les caractéristiques originales du porc de Piétrain ne permettent pas de le rattacher à d'autres races belges ou étrangères et excluent également 1'explication par un croisement. La conclusion de DERWA, à laquelle souscrit WILLEMS, est qu'il s'agit d'une mutation: "seule une variation brusque et héréditaire peut expliquer l'énorme développement des jambons, la largeur et l'épaisseur du carré, la finesse de l'ossature et un rapport graisse / viande des plus intéressants ". Une hypothèse similaire a été invoquée pour expliquer le " culard " bovinqui présente des analogies avec le phénomène Piétrain (voir LAUVERGNE et al., I963). Dans le cas du Piétrain en effet, divers travaux montraient clairement, dès le début des années 60 , la supériorité objective de cette race par rapport aux autres races européennes quant au pourcentage de muscle. Cette supériorité pouvait difficilement s'expliquer par une sélection continue sur un caractère polygénique, car, comme indiqué ci-dessus, la race était d'origine récente et, de plus, apparue spontanément et en dehors de toute organisation d'éleveurs jusqu'en r950. I1 s'agit en effet d'une race dont la genèse est tout à fait à l'opposé de celle du Landrace danois par exemple, qui résulte d'une volonté de planification émanant de 1'État (OLLIVIER, I976). L'hypothèse d'une origine mutationnelle de la race de Piétrain a été considérablement renforcée par les recherches de ces 20 dernières années. L'objet de cet article est précisément de présenter les arguments en faveur d'un déterminisme monogénique de l'hypertrophie musculaire qui est à l'origine du phénomène Piétrain.

\section{L'explication génétique du phénomène Piétrain}

Une difficulté commune à l'hypertrophie musculaire dans les espèces bovine et porcine est la définition précise du phénomène observé. La mise à l'épreuve de l'hypothèse d'un déterminisme monogénique du "caractère Piétrain ", qui fut décidée par 1'I.N.R.A. en I962, au domaine de La Haizerie (Calvados), était basée sur l'étude de deux critères. Le premier était une note visuelle de développement musculaire (OLLIVIER et LAUVERGNE, I967) inspirée d'un système appliqué aux bovins et le second, plus objectif, une combinaison linéaire de 6 mesures de carcasse (au poids vif d'abattage de $100 \mathrm{~kg}$ ) permettant de discriminer au mieux deux échantillons de race pure, un Large White et un Piétrain (OlLIVIER, I968). Le croisement entre ces deux races, en fait I o Piétrain $\times$ II ㅇ Large White, poursuivi jusqu'à l'obtention de $1 \mathrm{a} \mathrm{F}_{2}$ et des deux croisements en retour aux races parentales, mettait en évidence, pour les deux critères précédemment définis, des irrégularités de distribution simulant la ségrégation d'un gène à effet " majeur " présent à l'état homozygote chez le Piétrain et absent chez le Large White, 1'hétérozygote étant intermédiaire (fig. I et 2). Par ailleurs, une relation statistiquement significative était mise en évidence entre la note de conformation, donnée au sevrage vers $20 \mathrm{~kg}$ de poids vif, et la combinaison linéaire des 6 mesures de carcasse au poids de roo $\mathrm{kg}$. Cette convergence permettait d'expliquer comment la sélection sur la conformation au sevrage par les éleveurs belges de Piétrain pouvait être à l'origine du développement rapide de la race et de ses caractéristiques originales de composition corporelle. C'est en effet au sevrage que le sélectionneur traditionnel effectue le choix le plus sévère et, dans le cas du Piétrain, une sélection " à l'œil ", qui permettait de repérer un gène, devait être particulièrement efficace. Cependant, les effectifs d'animaux 


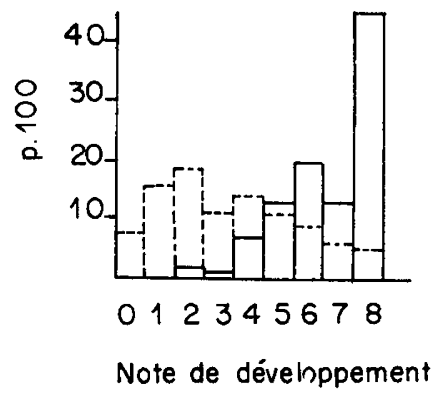

musculaire

FIG. I. - Distribution d'une note visuelle de développement musculaire dans les deux croisements en vetour $\mathrm{F}_{1} \times$ Piétrain et $\mathrm{F}_{\mathbf{1}} \times$ Large White (d'après OLLIVIER, 1968).

Distribution of a visual score for muscle development in the two backcrosses $\mathrm{F}_{\mathbf{1}} \times$ Pietrain and $\mathrm{F}_{1} \times$ Large White.

$\ldots \mathrm{F}_{1} \times$ Piétrain $(n=\mathrm{IoI}) \quad \ldots-\mathrm{F}_{1} \times$ Large White $(n=\mathrm{I1} 3)$

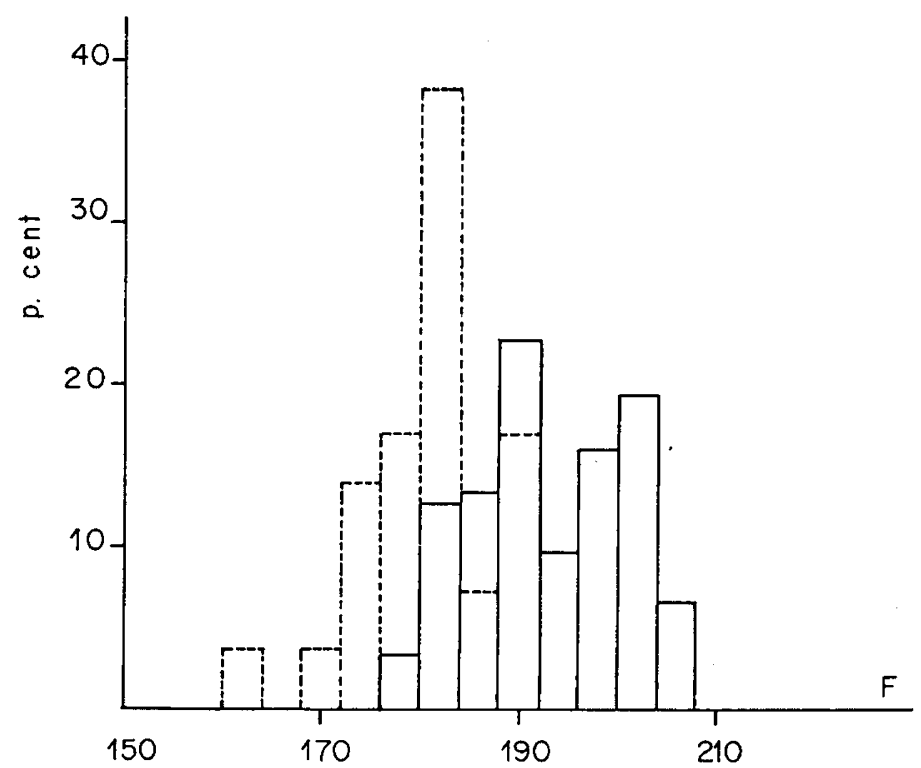

FIG. 2. - Distribution d'une fonction linéaire (F) de 6 mesures de carcasse dans les mêmes croisements que ceux de la figure I (d'après OLIIVIER, I968): $\mathrm{F}=$ Io $\mathrm{J}-0,8 \mathrm{C}+8 \mathrm{~L}+8 \mathrm{H}+$ $0,5 \mathrm{E}+9 \mathrm{P}$.

La signification des symboles des 6 variables et leurs unités sont données au tableau.

Distribution of a linear function (F) of 6 carcass measurements in the same crosses as those of figure $I$. Symbols and units for the 6 variables are given in Table.

$\mathrm{F}_{1} \times$ Piétrain $(n=3 \mathrm{I}) \quad \ldots \mathrm{F}_{1} \times$ Large White $(n=29)$

abattus en deuxième génération étaient insuffisants pour démontrer rigoureusement la ségrégation d'un gène et l'hypothèse monogénique n'était que suggérée. La conclusion de notre article de I 968 mérite peut-être d'être citée ici, pour son caractère prémonitoire, puisqu'elle soulignait la nécessité de "mettre au point un test vraiment spécifique de l'entité observée avant de poursuivre les études génétiques ". 


\section{Un test spécifique de l'entité Piétrain : 1'halothane}

L'expérience précédente devait en effet aboutir, quelques années plus tard, à la découverte d'un " test spécifique de l'entité Pietrain ». Ce fut le résultat d'une collaboration qui s'était établie entre le Département de Génétique animale à Jouyen-Josas et le Laboratoire de Recherches sur les Viandes de Theix. L'objectif visé était de rechercher des critères mesurables sur l'animal vivant qui pussent indiquer son aptitude à donner une viande de bonne qualité et qui permissent d'éliminer le défaut, fréquent en race de Piétrain, des viandes dites exsudatives. C'est ainsi qu'une relation statistiquement significative avait pu être établie entre la température musculaire mesurée in vivo sur la longe et le caractère exsudatif apprécié sur la coupe du jambon après abattage, à une température musculaire élevée correspondant une moins bonne rétention d'eau (PoTIER, I974). Sur le même troupeau expérimental Piétrain de Bourges, MonIN expérimentait en I974 un nouveau test, l'anesthésie à l'halothane, qui déclenchait chez certains animaux un ensemble de réactions connu sous le nom de syndrome d'hyperthermie maligne. Ce syndrome, caractérisé entre autres symptômes par une élévation très rapide de la température corporelle, avait été décrit depuis peu dans diverses espèces, dont surtout le Porc et 1'Homme. La réaction était du type "tout ou rien " et un déterminisme monogénique autosomal récessif pouvait l'expliquer parfaitement (Olinvier et al., I975). Cette hypothèse, dont nos résultats fournissaient la première preuve convaincante, a été depuis lors renforcée par de nombreux travaux. européens, dont ceux de Minkema et al. (I977), Smith et Bampton (I977), AndreSEN et JENSEN (I977) et SchwöRER et BLum (I977). En dehors de l'Europe, la même hypothèse, avancée par CHRISTIAN (I972), a été confirmée par MABRY (I977) et par McPheE et al. (I979), mais elle est rejetée par Wilisiams et al. (I975).

Il devenait dès lors tentant de rapprocher les résultats de l'expérience de croisement Piétrain $\times$ Large White (OLLIVIER, I968) — qui sera appelée expérience I - de ceux de notre premier test à l'halothane (OLLIVIER et al., I975) - qui sera appelée expérience 2 - et d'expliquer les irrégularités de distribution de l'expérience I par la ségrégation au locus de sensibilité à l'halothane ( $\mathrm{Hal}$ ) d'un gène normal provenant du Large White $\left(\mathrm{Hal}^{+}\right)$et d'un allèle de sensibilité provenant du Piétrain (Hals), que l'expérience 2 mettait en évidence mais dont l'existence n'était pas soupçonnée auparavant. Les arguments suivants à l'appui de ce rapprochement peuvent être avancés. (I) Le verrat Piétrain utilisé dans l'expérience $I$ mourut subitement, après avoir engendré la $F_{1}$, et les circonstances de cette mort laissent supposer qu'il était vraisemblablement sensible à l'halothane et donc de génotype $\mathrm{Hals}^{\mathrm{s}} / \mathrm{Hals}^{s}$. (III) Le système de notation du développement musculaire au sevrage, instauré en I 962 en vue de l'expérience $I$, a continué d'être appliqué par la suite, ce qui a permis de montrer qu'une étroite association, de nature génétique, existe ent:e 1'hypertrophie musculaire ainsi appréciée et le syndrome d'hyperthermie maligne. Les 3 observations suivantes viennent en effet appuyer cette hypothèse : la note de conformation au sevrage du père est liée au pourcentage de ses descendants sensibles à l'halothane (OLIIVIER et al., I975), la fréquence du gène de sensibilité à l'halothane augmente dans une lignée expérimentale sélectionnée sur la note de conformation (SELLIER, I980) et, dans les races porcines actuellement exploitées en France, la fréquence de l'anomalie va de pair avec le développement musculaire apparent au sevrage (OL, IVIER et al., I978). (III) Enfin, les mesures de carcasse retenues dans l'expérience I pour caractériser objectivement 1'hypertrophie musculaire du Piétrain sont parmi 


\section{TABLEAU}

Effet du type de croisement en $2^{\mathrm{e}}$ genération entre Piétrain et Large White $\left(D_{c}\right)$ et de la sensibilité à l'halothane dans la vace de Piétrain $\left(D_{s}\right)$ sur 6 variables de carcasse

Effect on 6 carcass measurements of the type of $2 d$ generation cross between Piétrain and Large White $\left(D_{c}\right)$ and of halothane sensitivity in the Piétrain breed $\left(D_{s}\right)$

\begin{tabular}{|c|c|c|c|}
\hline Variable $\left(^{(1)}\right.$ & Symbole & $\mathrm{D}_{c}\left(^{(2)}\right.$ & $\mathrm{D}_{s}\left(^{3}\right)$ \\
\hline 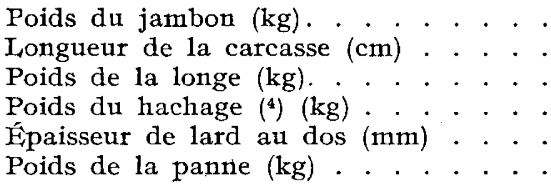 & $\begin{array}{l}\mathrm{J} \\
\mathrm{C} \\
\mathrm{L} \\
\mathrm{H} \\
\mathrm{E} \\
\mathrm{P}\end{array}$ & $\begin{aligned} & 0,19(*) \\
&-3,3(* *) \\
& 0,73\left(^{* *}\right) \\
& 0,26\left(^{*}\right) \\
&-0,8 \text { N.S. } \\
&-0,02 \text { N.S. }\end{aligned}$ & $\begin{aligned} & 0,50(* * *) \\
&-1,7(* * *) \\
& 0,63(* * *) \\
& 0,22(* *) \\
&-0,7 \text { N.S. } \\
&-0,14(* *)\end{aligned}$ \\
\hline
\end{tabular}

(1) Variables retenues, dans l'ordre indiqué, pour discriminer au mieux deux échantillons de race pure Piétrain et Large White (Variables selected in order to best discriminate between Large White and Piétrain : $J$ ham, $C$ carcass length, $L$ loin, $H$ belly, $E$ backfat thickness, $P$ leaf fat). OLIIVIER (I968).

(2) Différence entre les croisements en retour au Piétrain et au Large White (Difference between the Piétrain and Large White backcrosses). OLLIVIER (1968).

${ }^{(3)}$ Différence entre les Piétrain sensibles à l'halothane et les non sensibles (Difference between halothane positive and halothane negative Piétrain pigs). MoNin et al. (I976).

(4) Pour $\mathrm{D}_{c}$ il s'agit du poids de l'ensemble poitrine-hachage.

$(* * *)$ : Significatif au seuil de I p. I ooo $(\mathrm{P}<0.00 \mathrm{I})$.

$(* *)$ : Significatif au seuil de I p. roo $(\mathbf{P}<0.0 \mathrm{r})$.

$\left(^{*}\right)$ : Significatif au seuil de $5 \mathrm{p}$. roo $(\mathrm{P}<0.05)$.

NS : Non significatif (Not significant).

celles pour lesquelles les différences les plus significatives sont obtenues, d'une part dans la deuxième génération de l'expérience $I$, entre la $F_{2}$ et les deux croisements en retour et, d'autre part, dans l'expérience 2, entre les porcs sensibles et les non sensibles (tab1.). De plus, l'application de la fonction discriminante de l'expérience I (limitée aux 4 premières variables) à un échantillon de $I 76$ porcs Piétrain (incluant ceux de 1'expérience 2) étudié par MonIN et al. (I980) révèle des irrégularités de distribution qui peuvent s'expliquer, comme dans l'expérience I, par la ségrégation du gène de sensibilité à l'halothane (fig. 3). Cela permet de dire que la sensibilité à l'halothane correspond à un type Piétrain en quelque sorte exacerbé, caractérisé par une carcasse plus lourde, plus musclée et plus courte que le Piétrain normal.

\section{Un même gène détermine l'hypertrophie musculaire et l'hyperthermie maligne}

L'ensemble de ces résultats nous a conduit à postuler que le même gène est responsable de 1'hypertrophie musculaire et de 1'hyperthermie maligne (OLLIVIER et al., I975). Remarquons que la même hypothèse a été, simultanément et indépendamment, proposée par FREDEEN (I975). Il faut noter que le gène se comporte comme un récessif du point de vue de l'hyperthermie maligne alors que son effet est plutôt de type additif pour le développement musculaire (voir ci-dessous). Par ailleurs, 1'expression du gène quant à ces deux phénomènes est 

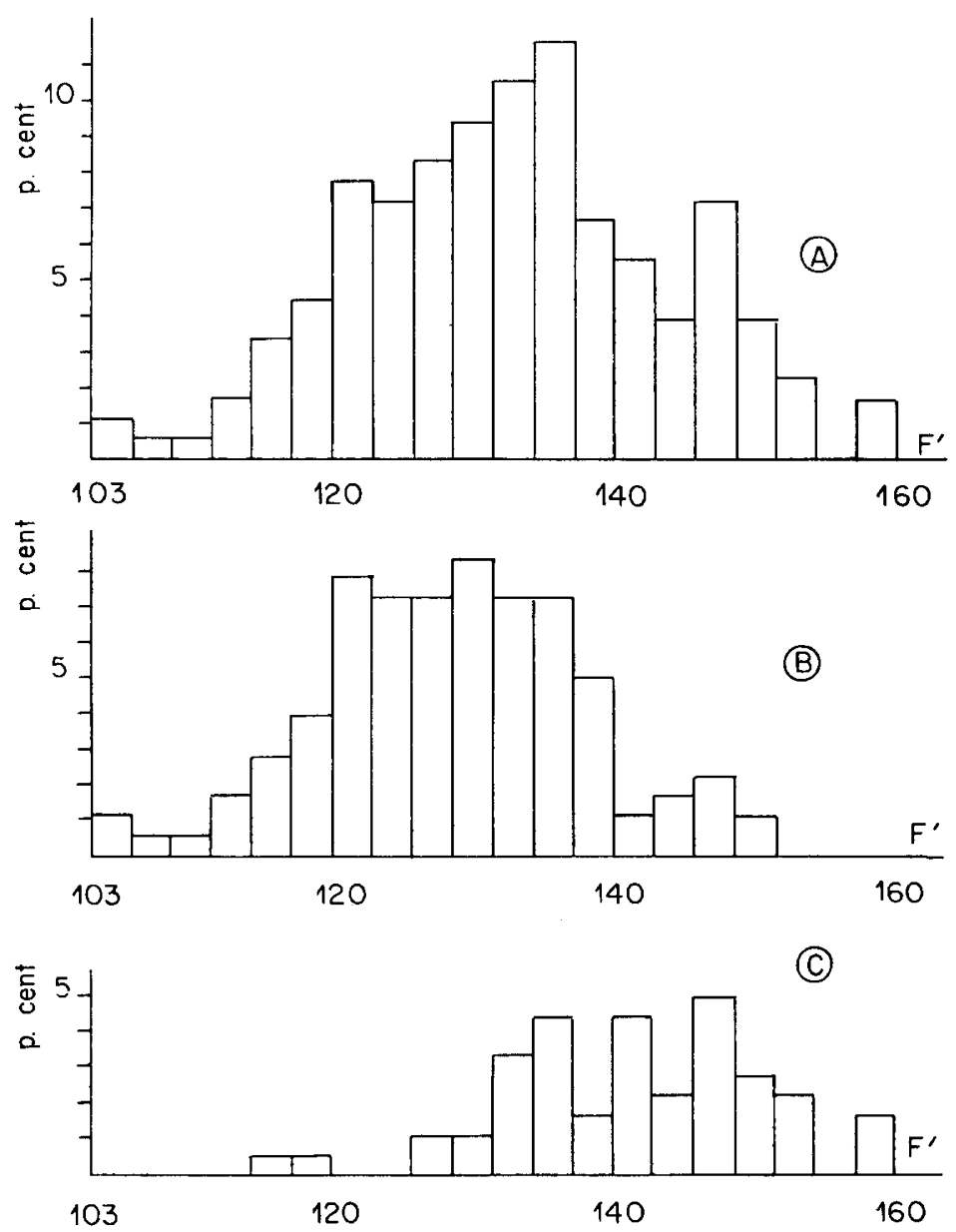

FIG. 3. - Distribution d'une fonction linéaive simplifiée $\left(\mathrm{F}^{\prime}\right)$ appliquée à des porcs de Piétrain soumis au test à l'halothane dans 2 experiences: $\mathrm{F}^{\prime}=10 \mathrm{~J}-0,8 \mathrm{C}+8 \mathrm{~L}+8 \mathrm{H}$. Les variables $J, C, L$ et $H$ sont corrigées pour les effets du sexe et de la date de l'expérience.

Distribution of a simplified linear function $\left(\mathrm{F}^{\prime}\right)$ applied to Piétrain pigs which weve halothanetested in 2 experiments. The four variables are corrected for the effects of sex and date of the experiment.
A : échantillon total (whole sample), $n=I 76$.
$\mathrm{B}$ : norl sensibles à l'halothane (halothane negative), $n=\mathrm{I} 20$
$\mathrm{C}$ : sensibles à l'halothane (halothane positive), $n=56$.

influencée par des facteurs de nature très différente, ce qui tend à atténuer l'étroitesse de la liaison phénotypique entre eux. Ainsi l'apparition du syndrome d'hyperthermie maligne dépend des modalités du test à 1'halothane (durée maximum, symptômes retenus pour décider l'arrêt du test, etc.). Cela explique sans doute, au moins partiellement, que le gène soit considéré comme ayant une " pénétrance " plus ou moins complète selon les auteurs. Quant au développement musculaire, l'appréciation visuelle qui en est faite dépend du développement général de l'animal. C'est ainsi que, si le pointage est effectué à poids fixe (par exemple 
vers $25 \mathrm{~kg}$ ), une corrélation significativement négative existe entre l'âge au pointage et la note de conformation (CARrouŕ, I976), une croissance lente défavorisant donc l'expression du développement musculaire.

Du point de vue de la nature exacte de la liaison génétique entre les deux phénomènes, une question reste cependant à préciser. L'association est-elle vraiment due à des effets pléiotropiques d'un même gène ou à une liaison génétique (" linkage ") entre des gènes situés à des locus rapprochés sur le même chromosome. Il est généralement difficile de trancher entre ces deux explications, surtout si la liaison génétique entre les locus concernés est très étroite. Cependant, les arguments dont nous disposons rendent l'hypothèse de pléiotropie plus plausible que celle d'un " linkage ". Le premier argument repose sur le fait que le gène de sensibilité à l'halothane est défavorisé par la sélection naturelle, puisqu'il entraîne une mortalité nettement accrue et aussi une prolificité des truies probablement diminuée (voir WEBB et JoRDAN, I978). Son maintien à des fréquences élevées ne peut donc s'expliquer que par une sélection artificielle favorable au gène, ce qui implique qu'il ait un effet pléiotropique sur des caractères recherchés par les éleveurs. Le fait que la charnure soit un caractère visible très apprécié de longue date par les éleveurs belges amène à supposer que le gène exerce un effet pléiotropique favorable sur la charnure (OLLIVIER et al., I975). Pour que 1'hétérozygote $\left(\mathrm{Hal}^{+} / \mathrm{Hal}^{8}\right)$ soit globalement favorisé par rapport aux deux homozygotes, il faut supposer en outre qu'il possède un avantage du point de vue de la charnure par rapport à l'homozygote normal $\left(\mathrm{Hal}^{+} / \mathrm{Hal}^{+}\right)$, ce qui implique que le gène ne soit pas récessif dans son action sur le développement musculaire. Nos premières observations, qui allaient dans ce sens (OLLIVIER et al., I975), ont été ultérieurement confirmées par divers travaux, dont ceux de ANSAY et OLLIVIER (I978) et EIKELENBOOM et al. (I980). D'autres arguments en faveur de la pléiotropie sont fournis par l'étude des liaisons génétiques entre le locus $\mathrm{Hal}$ et d'autres polymorphismes (voir paragraphe suivant). Enfin, une homologie entre espèces mérite d'être signalée à l'appui de la pléiotropie : l'hyperthermie maligne chez 1'Homme est également parfois associée à un fort développement musculaire (voir GoRDon et al., I973).

\section{Localisation du gène de sensibilité à l'halothane}

Le point de départ de ces travaux fut la découverte en I976 d'une association entre 1'hyperthermie maligne et des polymorphismes biochimiques à deux locus, le locus $H$ de groupe sanguin (RASMUSEN et Christian, I976) et le locus de la phosphohexose isomérase $(P H I)$, qui est une enzyme du globule rouge (JøRGENSEN et al., I976). On pouvait même conclure de ces derniers travaux que le locus de la $P H I$ était lui-même responsable de l'hyperthermie maligne (JøRGENSEN, I978), puisque tous les animaux sensibles dans le Landrace danois étaient homozygotes $(B B)$ au locus $P H I$. Cette hypothèse dut cependant être rapidement abandonnée à la suite des travaux de 1'I.N.R.A. sur des lignées Piétrain, desquels il ressortait que la liaison n'est pas absolue entre l'hyperthermie maligne et le locus $P H I$ (ANSAy et OliLIVIER, I978; GUÉRIN et al., I978). D'autre part, ANDRESEN (I97I) avait montré l'existence d'un groupe de liaison incluant dans cet ordre les locus $P H I, H$ et 6-PGD (6-Phosphogluconate déshydrogénase, une autre enzyme du globule rouge). Le locus $H a l$ se situe très près de $P H I$ (environ à 0,5 centimorgans) et l'ordre probable de ces trois locus est $H a l-P H I-H-6-P G D$. La longueur de ce segment de chromosome, estimée à 6 centimorgans (entre $P H I$ et $6-P G D$ ) par 
ANDRESEN (I970 $a$ et $b$ ) et par OrShI et ABE (I979), doit probablement être révisée en baisse sur la base de résultats récents obtenus dans les lignées Piétrain de I'I.N.R.A. (GUÉRIN, communication personnelle).

L'étude des associations observées entre les locus $\mathrm{Hal}$ et $P H I$ contribue par ailleurs à résoudre le " dilemme pléiotropie-linkage " précédemment évoqué. L'argumentation qui va être développée ici repose sur des considérations théoriques de génétique des populations (THOMson, I977) que confirment par ailleurs des observations sur diverses races et lignées porcines (GuḱRIN et al., I979). Considérons deux locus $A$ et $B$, l'un $A$ exerçant un effet sur un caractère quantitatif, l'autre $B$ étant " neutre ". S'il y a une association gamétique entre les deux locus (ou déséquilibre de "linkage "), le locus $B$, quoique neutre, apparaît comme ayant un effet sur le caractère auantitatif. D'autre part, une sélection exercée au locus $A$ peut engendrer, en vertu d'un effet d'entraînement de $A$ sur $B$, un déséquilibre entre les locus $A$ et $B$ s'ils sont étroitement liés. Mais, pour que ce déséquilibre se maintienne à un niveau important, il faut que la sélection favorise l'hétérozygote au locus $B$. En particulier, si la sélection au locus $A$ est de type additif, la valeur sélective de 1'hétérozygote étant intermédiaire entre celle des deux homozygotes, le déséquilibre entre les deux locus ne se maintient pas et le locus $B$ ne peut pas alors manifester d'effet apparent sur le caractère considéré. Si les éleveurs belges avaient donc sélectionné pour un gène d'hypertrophie musculaire (à effet additif) distinct du gène de l'hyperthermie maligne (supposé neutre), il eût été peu probable de voir apparaître une association entre les deux phénomènes, en dépit de la liaison étroite entre les deux locus. L'existence de cette association tend donc à écarter 1'hypothèse de deux locus distincts. Notons d'ailleurs que, dans une lignée expérimentale où la sélection pratiquée tend à augmenter la fréquence du gène Hals (les homozygotes $\mathrm{Hals}_{\text {/ }}$ Hals étant favorisés), le déséquilibre entre ce locus et le locus " neutre " $P H I$ est très faible, alors que ce déséquilibre est important dans les populations porcines de type Piétrain où, dans l'hypothèse d'un seul locus, les hétérozygotes $\mathrm{Hal}^{+} / \mathrm{Hal}^{s}$ sont globalement favorisés, pour les raisons données plus haut. Cela confirme les prévisions théoriques précédentes concernant les effets de ces deux types de sélection (Gú́RIN et al., I979). Cette explication de l'effet apparent du locus $P H I$ sur le développement musculaire (ou l'hyperthermie maligne) vaut également pour le locus $H$ de groupe sanguin auquel RASMUSEN et ChRISTIAN (I976) ont attribué, sans doute à tort, un effet physiologique propre quant à la réaction à l'halothane.

\section{Autres manifestations du phénomène d'hypertrophie musculaire}

La simplicité, le faible coût et le caractère très répétable du test à 1'halothane expliquent le succès de cette technique qui est en passe de devenir un outil courant dans la pratique de la sélection porcine. Si on peut admettre, comme on vient de le voir, que ce test met en évidence un gène " majeur » de développement musculaire, tous les caractères qui ont été étudiés en relation avec ce test peuvent également être considérés comme associés à 1'hypertrophie musculaire.

Les éleveurs de porcs de Piétrain avaient noté très tôt chez les animaux à musculature hypertrophiée une fréquence élevée des cas de mort subite consécutifs à des contraintes de nature très variée, telles que le transport, la saillie, les manipulations, etc. Ces porcs avaient la réputation d'être "cardiaques ". Il semble maintenant assez bien établi que seuls les porcs sensibles à l'halothane sont soumis 
à ce risque et que le même mécanisme conduit aux deux syndromes de stress (PSS dans la terminologie anglaise) et d'hyperthermie maligne (MHS). La mortalité au cours de l'engraissement et du transport à l'abattoir est Io fois plus élevée chez les porcs sensibles à 1'halothane que chez les porcs normaux dans le Landrace hollandais (EIKELENBOOM et al., I978). Notons ici qu'il faut distinguer ce syndrome de stress, le plus souvent fata1, des phénomènes généraux de stress. DANTZER et MORMÈDE (I978) ont ainsi montré, sur la base d'une épreuve d'évitement continu réalisée dans une cage à double compartiment, que le porc de Piétrain s'adapte mieux que le Large White à des situations aversives, et les porcs sensibles à l'halothane mieux que les porcs normaux, quoique moins nettement. Autrement dit, dans des conditions de stress modéré, les porcs sensibles à l'halothane sont au moins aussi résistants que les porcs normaux.

Comme indiqué ci-dessus, le test à l'halothane a été d'abord étudié en tant que critère in vivo de qualité de la viande, et c'est en vue de prédire le défaut des viandes dites exsudatives (PSE dans la terminologie anglaise) qu'il fut initialement proposé par EIKELENBOOM et Minkema (I974). De nombreux travaux ont par la suite confirmé que la sensibilité à l'halothane est associée à une viande pâle, flasque, à faible pouvoir de rétention d'eau et caractérisée par une chûte rapide de $\mathrm{pH}$ post-mortem. De nombreuses caractéristiques sanguines, dont l'enzyme créatine-kinase, sont également affectées (Monin et al., I979). L'effet du locus Hal est vraisemblablement additif ici comme pour le développement musculaire (ANDRESEN et JENSEN, I978). Il faut noter cependant l'existence d'une interaction très marquée entre le génotype et les conditions d'abattage, comme cela a été montré, en particulier, par MonIN et al. (I980). Les différences de qualité terhnologique du jambon obtenues entre les animaux sensibles et les normaux son' d'autant plus marquées que l'animal est plus proche de l'état de repos au moment ce l'abattage. Par contre, le défaut opposé au précédent, caractérisé par l'aspect sombre, ferme et sec de la viande (DFD en anglais), ne semble pas lié à la sensibilité à 1'halothane. Ainsi, la glycogénolyse musculaire post-mortem, qui produit les viandes exsudatives, aurait une base physiologique différente de celle de la mobilisation du glycogène musculaire in vivo, phénomène responsable des viandes sombres (MONIN et al., I980).

C'est donc le même mécanisme biochimique qui aboutit soit à la manifestation du syndrome d'hyperthermie maligne, soit à la production de viande exsudative dans certaines conditions d'abattage. L'étude de ce mécanisme a donné lieu à de nombreux travaux qui tendent à indiquer une régulation anormale des mouvements $\mathrm{du} \mathrm{Ca}^{++}$dans la fibre musculaire. Le fonctionnement du réticulum sarcoplasmique a été mis en cause, mais des résultats contradictoires ont été obtenus à ce sujet (CAMPION et al., I979). Les récents travaux de CHEAH et CHEAH (I978) indiquent que le trouble se situerait plutôt au niveau des mitochondries du muscle. Les résultats de ces auteurs suggèrent en effet qu'il peut exister des différences dans la structure ou dans le fonctionnement des membranes mitochondriales entre les porcs sensibles à l'halothane et les porcs normaux. D'autres membranes seraient également affectées, en particulier celle des globules rouges, comme le montre le test osmotique (KING et al., I976).

Les études qui viennent d'être passées en revue tendent à privilégier les aspects liés au "stress" et à la qualité des viandes. Mais le mécanisme biologique qui conduit à l'hypertrophie musculaire elle-même est loin d'être élucidé. Le phénomène a été bien décrit au niveau musculaire par Dumont et al. (I969) et Dumont et Schmitr (1970). L'hypertrophie est due à l'augmentation de la taille des fibres musculaires, elle s'accompagne de la présence de fibres " géantes " et, 
si on considère la totalité de la section d'un muscle, la trame conjonctive apparaît plus fine et plus distendue chez les individus (et les muscles) hypertrophiés. Cette dernière observation est confirmée par des dosages d'hydroxyproline, acide aminé caractéristique du collagène, dont le taux est inférieur de I2 p. Ioo chez le Piétrain par rapport au Large White (SELLIER et BOCCARD, I97I), ce qui traduirait un déficit en collagène plus ou moins généralisé dans l'organisme. Dans l'état actuel des recherches, si l'hypertrophie musculaire du Porc et l'ensemble des autres phénomènes qui lui sont associés paraissent se rattacher à l'action pléiotropique d'un gène unique, le lien biologique entre les diverses manifestations phénotypiques de ce gène, qui serait l'action " primaire " du gène, reste encore à établir. Il faut aussi mentionner qu'en dehors du locus $H a l$ une variation génétique importante existe, tant pour le développement musculaire que pour la qualité de la viande. L'étude des races où le gène $\mathrm{Hal}^{\mathrm{s}}$ a une fréquence négligeable, comme le Large White, le montre clairement, tout comme la comparaison entre les homozygotes $\mathrm{Hal}^{\mathbf{s}} / \mathrm{Hal}^{s}$ de différentes races.

$$
\text { Reçu pour publication en janvier } 198 \mathrm{I} \text {. }
$$

\section{Remerciements}

Au Pr HANSET (Ếcole vétérinaire, Bruxelles) pour avoir bien voulu lire le manuscrit.

\section{Summary}

\section{Genetic determination of Muscular Hypertrophy in the Pig}

Very early, the origin of the Belgian pig of Piétrain has been a matter of speculations. Several Belgian authors have put forward a hypothesis according to which the Piétrain breeders would have detected and selected for a mutation. Indeed, the exceptional muscle development of the breed cannot easily be explained by selection for a quantitative polygenic trait. A crossbreeding experiment was conducted in France, some fifteen years ago, in order to test the above hypothesis. In that experiment, muscle hypertrophy was assessed in two different ways, namely by a visual score for conformation at weaning ( $20 \mathrm{~kg}$ liveweight) and by a combination of several carcass measurements at Ioo $\mathrm{kg}$ liveweight. In both cases the results strongly suggested the existence of a major gene responsible for muscle hypertrophy in the Piétrain pig. Later on the evidence found for a monogenic determination of the halothane-induced malignant hyperthermia syndrome (MHS) and the narrow links between MHS and muscularity naturally led to postulate that a single pleiotropie gene might be responsible for both phenomena. Thus, halothane inhalation may be considered as a specific test for the "Piétrain entity " previously defined. The relationships found between the MHS gene and three different biochemical polymorphisms loci allow to locate the gene, rather precisely, within a " chromosome map " about 6 centimorgans long. The incidence of MHS on meat quality has been very intensively investigated in the recent years and the underlying biological mechanism might be a defect of the mitochondrial membrane affecting the $\mathrm{Ca}++$ transport system in the muscle. However, the biological mechanism leading to muscular hypertrophy itself still remains unexplained.

\section{Références bibliographiques}

Addis P. B., Burris W. I., ANTonik A., 1976. Muscle characteristics and blood creatinine kinase in stress-susceptible and stress-resistant breeds. Proc. 4th Intern. Pig Vet. Soc. Congress, Ames, Iowa ( $\left.\mathrm{T}_{7}\right)$.

ANDRESEN E., I970a. Linkage between the $\mathrm{H}$ and 6-PGD loci in pigs. Acta vet. scand., 11, I36-I37.

ANDRESEN E., r97ob. Close linkage between the locus for phosphohexose isomerase (PHI) and the $\mathrm{H}$ blood group locus in pigs. Anim. Blood Grps biochem. Genet., 1, I7I-172. 
ANDRESEN E., I97I. Linear sequence of the autosomal loci PHI, H and 6-PGD in pigs. Anim. Blood Grps biochem. Genet., 2, II9-120.

ANDRESEN E., JENSEN P., I977. Close linkage estabiished between the Hal locus for halothane sensitivity and the PHI (phospohexose isomerase) locus in pigs of the Danish Landrace breed. Nord. Vet. Med., 29, 502-504.

ANDRESEN E., JENSEN P., I978. Evidence of an additive effect of alleles of the Hal locus on the KK index for porcine meat quality. Nord. Vet. Med., 30, 286-288.

ANSAY M., OLI,IVIER L., I978. Créatinine plasmatique et sensibilité du porc au syndrome d'hyferthermie maligne. Relations avec deux enzymes du globule rouge (PHI et 6-PGD). Ann. Génét. Sél. anim., 10, 9-16.

Campion D. R., Eikelendoom G., Cassens R. G., I979. Effect of halothane on isometric contractile propertics of muscle from stress-susceptible and stress-resistant pigs. Res. Vet. Sci., 27, I $16-\mathbf{1} \mathbf{8}$.

CARRoué J. P., I976. Étude des relations entre la conformation appréciée sur l'animal vivant, la vitesse de croissance et l'épaisseur du lard dorsal chez le porc de Piétrain. Rapport de stage I.N.R.A. Génétique animale, 84 p. (polycopié).

CHEAH K. S., CHEAH A. M., 1978. Calcium movements in skeletal muscle mitochondria of malignant hyperthermic pigs. Febs Lett., 95, 307-310.

ChrIstran L. L., I972. A review of the role of genetics in animal stress susceptibility and meat quality. In Cassens R., Giesler F., KolB Q., Proc. Pork Qual. Symp., 9i-I I5, Univ. Wisconsin, Madison, Wisconsin.

DANTZER R., MORMĖDE P., I978. Behavioural and pituitary-adrenal characteristics of pigs differing by their susceptibility to the malignant hyperthermia syndrome induced by halothane anesthesia. I. Behavioural measures. Ann. Rech. Vet., 9, 559-567.

Dumont B. L., Schmrit O., I970. Anatomie microscopique comparée du tissu musculaire squelettique de porcs Large White et Piétrain. Ann. Génét. Sél. anim., 2, 381-391.

Dumont B. L., SchmitT O., Roy G., I969. Développement musculaire comparé de porcs Piétrain et Large White. Rec. Méd. Vét., 145, 937-948.

Eikelenboom G., Minkema D., r974. Prediction of pale, soft, exudative muscle with a nonlethal test for the halothane-induced malignant hyperthermia syndrome. Neth. J. Vet. Sci., 99, 42 I-426.

EIKElenboom G., Minkema D., VAN El,dik P., Sybesma W., I978. Production characteristics of Dutch Landrace and Dutch Yorkshire pigs as related to their susceptibility for the halothaneinduced malignant hyperthermia syndrome. Livest. Prod. Sci., 5, 277-284.

Eikfinenboom G., Mrnkema D., Van Eldik P., Sxbesma W., 1980. Production traits of Dutch Landrace pigs as related to their genotype and phenotype for the halothane-induced malignant hyperthermia syndrome. Livest. Prod. Sci., 7 (sous presse).

FREDEEN H. T., I975. Future aspects in breeding a modern meat type pig. In Fetskrift til Hjalmar Clausen, 49-7I, Det. Kgl. Landhusholdningsselskab, Copenhague.

GORDON R. A., BRITT B. A., KALOW W., I973. International Symposium on Malignant Hyperthermia. Charles C. Thomas, Springfield, Illinois.

GuÉrin G., OLLIVIER I., SELI,IER P., I978. Note sur les déséquilibres de linkage entre les locus Hal (hyperthermie maligne), PHI et 6-PGD dans deux lignées Piétrain. Ann. Génét. Sél. anim., 10, I25-I29.

GứRIN G., OLLIVIER I., SELLIER P., I979. Effet d'entraînement d'un gène sélectionné et association gamétique (déséquilibre de "linkage") : l'exemple de deux locus étroitement liés chez le Porc, Hal (sensibilité à l'halothane) et PHI (phosphohexose isomérase). C. R. Acad. Sci. Paris, série $D, 289$, I53-ז 56.

JøRGENSEN P. F., x978. Halothane sensitivity, the $\mathrm{H}$ blood group system and phosphohexose isomerase (PHI) in pigs. A linkage of physiological importance. Acta Vet. Scand., 19, 458-460.

Jørgensen P. F., HyldgaARd-Jensen J., Moustgaard J., Eikelenboom G., i976. Phosphohexose isomerase (PHI) and porcine halothane sensitivity. Acta Vet. Scand., 17, $370-372$.

KING W. A., Or,IVIER L., BASRUR P. K., r976. Erythrocyte osmotic response test on malignant hyperthermia susceptible pigs. Ann. Génét. Sél. anim., 8, 537-540.

I,AUVERgne J. J., VisSAC B., Perramon A., I963. Étude du caractère culard. I. — Mise au point bibliographique. Ann. Zootech., 12, I33-I 56.

MABRY J. W., I977. Porcine stress syndrome: inheritance, prediction and performance characteristics. Ph. D. Iowa State University, ro2 p.

MCPHEE C. P., TAKKEN A., D'ARCY K. J., I979. Genetic variation in meat quality and the incidence of malignant hyperthermia syndrome in Large White and Landrace boars. Aust. J. Exp. Agric. Anim. Husb., 19, 43-47. 
MINKEMA D., EIKELENBOOM G., VAN Eldik P., I977. Inheritance of MHS-susceptibility in pigs. In Proc. Third Int. Conf. on Production Disease in Farm Animals, 203-207, Pudoc, Wagenirigen, Pays-Bas.

MONIN G., OLLIVIER I., SELLIER P., I976. Étude du syndrome d'hyperthermie maligne chez le porc de Piétrain: premiers résultats. In Journées de la Recherche Porcine en France 1976, 229-239, Institut technique du Porc, Paris (Ann. Zootech., 25, 447-448, abstr.)

MONIN G., SELIIER P., OLLIVIER L., I979. Étude de quelques caractéristiques sanguines de populations porcines françaises. Relations avec Je syndrome d'hyperthermie maligne. $A n n$. Rech. Vèt., 10, 533-547.

Monin G., Selifier P., Ollivier L., Goutefongea R., Girard J. P., i98o. Carcass characteristics aud meat quality of halothane negative and halothane positive Piétrain pigs. Meat Sci. (sous presse).

Nelson T. E., Jones E. W., Henrickson R. L., FALK S. N., KERP D. D., I974. Porcine malignant hyperthermia: observations on the occurrence of pale, soft, exudative musculature among susceptible pigs. Am. J. Vet. Res., 35, 347-350.

OIshi T., ABE T., I979. Polymorphism of PHI, 6-PGD, PGM and ADA enzymes in porcine red cells. Bull. Nat. Inst. Anim. Ind., 35, 9-18.

OLLIVIER L., I968. Étude du déterminisme héréditaire de l'hypertrophie musculaire du porc de Piétrain. Ann. Zootech., 17, 393-407.

Or.trvier I., 1976. Évolution des méthodes de sélection du Porc. Ethnczootechnie, 16, 40-53.

Ollivier I., I.AUVERGNe J. J., I967. Étude du déterminisme béréditaire de l'hypertrophie musculaire du porc de Pietvain: premiers résultats. Ann. Méd. Vét., 111, Io4-Io9.

Olirvier L., Seilier P., Monin G., I975. Déterminisme génétique du syndrome d'hyperthermie maligne chez le porc de Piétrain. Ann. Génét. Sél. anim., 7, r59-166.

Olitivier L., SELIIER P., Monin G., I978. Fréquence du syndrome d'hyperthermie maligne dans des populations porcines françaises; relation avec le développement musculaire. $A n n$. Génét. Sél. anim., 10, I9I-208.

Potier D., I974. Possibilités d'amélioration génétique de la qualité de la viande chez le Porc. Rapport de stage I.N.R.A.-Génétique animale, 35 p. (polycopié).

RASMUSEN B. A., Christian L. L., I976. H blood types in pigs as predictors of stress susceptibility. Science, 191, 947-948.

SchwöRER D., BLUM J., 1977. Blasses, wässeriges Schweinefleisch: Voraussage mangelhafter Fleischbeschaffenheit bereits am lebenden Tier. Der Kleinviehzüchter, 25, 64I-650.

SFLIIFR P., I980. Preliminary results of a selection experiment for muscle hypertrophy in the Piétrain breed. Séminaire C.E.E. "Hypertrophie musculaire chez les bovins ", Toulouse, IO-I 2 juin I980.

SELIIER P., BOCCARD R., I97I. Étude du taux d'hydroxyproline d'un muscle et de ses relations avec certaines caractéristiques de composition corporelle dans les races Lavge White et de Piétrain. Ann. Génét. Sél. anim., 3, 443-448.

Smith C., BAmpton P. R., 1977. Inheritance of reaction to halothane anaesthesia in pigs. Genet. Res., 29, 287-292.

Thomson G., I977. The effect of a selected locus on linked neutral loci. Genetics, 85, $753-788$.

WEBB A. J., JoRdAN C. H. C., I978. Halothane sensitivity as a field test for stress-susceptibility in the pig. Anim. Prod., 26, I57-I68.

Willems A. E. R., I960. Origine et évolution du porc belge de Piétrain. In La race porcine belge Piétrain. Service des informations agricoles, Bruxelles.

Wilirams C. H., I.ASLEY J. F., MUhrer M. E., HeDrick H. B., I975. Relationship between fulminant hyperthermia and the porcine stress syndrome. J. Anim. Sci., 41, 261 (abstr.). 